\title{
CONTRIBUCION AL ESTUDIO DE LOS GUANCHISMOS EN LAS ISLAS CANARIAS
}

Durante mi estancia en Tenerife con ocasión de mi participación en las enseñanzas de la Facultad de Filosofla y Letras de la Universidad de La Laguna como profesor de Lingüística románica ( $\mathrm{r}^{\circ}$ de octubre de 1950 hasta fines de febrero de I95I) a fuer de apasionado cultivador de la Dialectologia, no pude desprenderme del problema más cautivador que se presenta en el campo de los estudios canarios, es decir, de la cuestión de los elementos indígenas supervivientes enl el habla de Canarias.

Mucho se ha hecho en los últimos quince años en este sentido. Nuevos materiales nos han suministrado los trabajos de don J. Álvarez Delgado, investigador infatigable, de don M̀ax Steffen y de don J. Régulo Pérez, precisando las acepciones de las voces, determinando $\in 1$ territorio de su empleo y estudiando las relaciones con el léxico castellano y galaico-portugués. Ocupa un puesto de honor en esta laudable actividad la Revista de Historia que se publica bajo la inteligente dirección de Elías Serra Ráfols, decano de la Facultad de Filosofía y Letras de la Universidad de La Laguna (Tenerife).

Falta todavía un riguroso método científico para la identificación de los dialectalismos canarios con los de las lenguas de Africa del Norte. Han sido repetidamente objeto de acerbas críticas los trabajos de J. Álvarez Delgado, a quien se ha reprochado su hambre de grandes novedades, su negación e ignorancia de los hechos, sus dudosas doctrinas lingüísticas y se le ha calificado de defensor a ultranza de guanchismos 
inexistentes ${ }^{1}$. Son muy fantásticas, por no decir absurdas, las etimologías que el señor Álvarez propuso para algunos nombres dados por los pastores de la isla del Hierro a sus ganados, relacionándolos con el hebreo y el árabe (Rev. de Hist. XII, I946, I6I-I64). Desconociendo las lenguas peninsulares en sus variedades dialectales y regionales, ha atribuido en "Miscelánea guanche" (I942) a la antigua lengua indfgena voces como burgaño 'ratoncito', mago 'campesino inculto', lindes 'nidal', bucio 'caracol marino', taboco 'concavidad', totizo 'cogote', todas ellas de indudable origen peninsular (español dialectal, gallego, portugués) ${ }^{2}$. Igualmente las audaces conexiones de voces canarias con lenguas de Africa del Norte y hasta con el vascuence, propuestas por don J. Wölfel ${ }^{3}$, me parecen desprovistas de toda probabilidad 4 . Son dignas de más confianza algunas comparaciones con dialectos beréberes presentadas por don W. Giese ${ }^{5}$; aunque en sus atribuciones hay mucho que no me convence.

Es mi opinión que, sin ser africanista especializado en las lenguas del grupo beréber-hamítico, es muy osado intentar conexiones que merezcan confianza. I,imitémonos, nosotros

1 Véase J. Rígui.o Pírez en Rev. de Hist. XIV, 1948, 478-489; Max Stefren, Reu. de Hist. IX, 1943, I34-I4I.

- Sobre elementos portugueses y americanos en el habla canaria, véase MI. L. WAGNER en su reseña del Léxico de Gran Canaria de Millares ( $R F E, \mathrm{XII}, 1925,78$ siguientes).

3 Die Hauptprobleme IVeissafrikas en Archiv für Anthropologie, XXVII, 1942, 133-140. Véanse también las explicaciones intentadas por Wölfel sobre nombres de lugares y de personas en el glosario añadido a su edición de LEONARDO TORRIANI, Die kanarischen Inseln, Lipsia, 1940.

- Damos aquil un solo ejemplo. Ia voz gilbarbera (gibarbera) 'Ruscus androgynus' no tiene nada que ver con el hausa awarwar $\hat{o}$ respecie de convolvulus (Wör.FEL, Die Hauptprobleme Weissafrikas, página 134: véase también Alvarkiz Disloado en Rev. de Hist. X, 1944, 243-244), sino que es idéntica al gallego-portugués gilbarbeira (gilbardeira), xilbarbeira umata espinosa", "brusco"; véase STEFEEN, Revista de Hist. XI, 1945, I48-157.

- Acerca del carácter de la lengua guanche eu Rev. de Hist. XV, I949, I 88-203. 
romanistas, a presentar a los africanistas los materiales canarios con la mayor posible determinación desde el punto de vista fonético y semántico y dejemos a ellos la tarea de un análisis lingüístico. Eso es lo que me propongo con este artículo. Presento aquí una colección de voces usadas en los dialectos callarios de hoy que, si no me equivoco, no se encuentran en las lenguas y los dialectos de la península hispánica, voces que con mucha probabilidad deben de relacionarse con las lenguas africanas 1.

He podido recoger mis materiales personalmente en varios pueblos de Tenerife. Respecto a las otras islas del Archipiélago pude realizar unas encuestas con mis alumnos de Gran Canaria, La Palma y La Gomera; otras con alumnos del Seminario de La Laguna nativos de Lanzarote y Fuerteventura. Ha sido particularmente fecunda una encuesta que, merced a un permiso especial, pude llevar a cabo en el cuartel de artillería de Santa Cruz de Tenerife, donde fueron puestos a mi disposición algunos soldados de varios pueblos de La Gomera y de Hierro, hijos de pastores, bastante inteligentes y muy entendidos en el léxico rural y pastoril.

Es para mi un grato deber dar aquí mis más expresivas gracias a todas las autoridades y personas que, durante mi estancia en Tenerife, me fueron generosas con su ayuda y su amabilidad: el Gobernador Civil de Tenerife don Luis Rosón, el rector de la Universidad don Alberto Navarro González y el decano de la Facultad de Filosofía y Letras don Elías Serra Ráfols. Me han suministrado útiles consejos y apreciable colaboración mis amigos don Juan Régulo Pérez, don Max Steffen, que ha contribuído con preciosas notas a este artículo, y mi alumuo don Antero Simón ${ }^{2}$.

- No me ocupo en este artículo de las palabras de la antigua lengua guanche, transmitidas a nosotros por los historiadores del tiempo de la conquista y que ell el habla de hoy no sobrevivan.

2 Indico la bibliografía y abreviaturas empleadas en este articulo: Armas Ayala: AlfoNso DE ARikas AYALA, Pequeño vocabulario de 
Vocabulario ${ }^{1}$.

*ajergo "vara con pelota de tela en su extremo para revolver el trigo en el tostador' (MG, I, pág. I6).--Verificado con la misma forma y en el mismo sentido en Güimar (Ten.) y en Agulo, Hermigua, Valle Gran Rey (La Gomera). En la

voces canarias, en Tradiciones populares, tomo I, palabras y cosas, prólogo del Dr. E. Serra Ráfols, I, Laguna de Tenerife, r944.

DMLE.-Diccionario manual e ilustrado de la lengua española de la

Real Academia Española, Madrid, I927.

F. = Fuerteventura.

GC. = Gran Canaria.

Go. = Gomera.

Lanz. = Lanzarote.

Lugo: SEBAstiAn DE I,UGo, Colección de voces y frases provinciales de Canarias. Edición, prólogo y notas de José Pérez Vidal. La Iaguna de Tenerife, 1946.

MG.-J. Alvariz Delgado, Miscelánea guanche. Parte I. Benahoare. La Laguna de Tenerife, 1942.

afillares: L. y A. Milladis CuBAs, Como hablan los canarios, Las Palmas, I932.

Reyes: JUAN REYES MARTIN, Sevie de barbarismos, solecismos, aldeanismos y provincialismos que se refieren especialmente al vulgo tinerfeño, Santa Cruz de Tenerife, s. a.

RDTP.-Revista de Dialectologta y Tradiciones populares (Madrid).

RH.-Revista de Historia (La Laguna de Tenerife).

Steffen: MAX STEFFEN, Lexicologia canaria, en RH, XI, 1945, I30-I77.

Steiger: A. STEIGER, Contribución a la fonética del hispano-árabe y de los arabismos en el ibero-románico, Madrid, I932.

Ten. = Tenerife.

TP.-Tradiciones populares. Parte I. Palabras y cosas. Colección de enzsayos y notas de folklore canario. Prólogo del Dr. I. Serra Ráfols, La I,aguna de Tenerife, 1944.

1 Designo con un asterisco las voces que no se encuentran en las colecciones de MIILLARISS y de I,Ugo.-He incluido ell este vocabulario algunas palabras que, sin ser guanclismos, han podido ser introducidas en la lengua guanclie en virtud de relaciones directas con el continente africano (arabismos). 
isla de Hierro (La Frontera, Mocanal, El Piuar) se dice juercan.

*algaritofe, alcalitofe, algaritopa, calitofe 'boca de dragón', "Dracocephalum canariense" ('Tenerife).-En Güimar (Ten.) algaritofe; en El Pinar (Hierro) caletoce. Formas recogidas por don Juan Régulo Pérez en La Palma son garitope y garitopa (RH, XIII, I947, I93).

*alparroba 'clase de siempreviva', "Sempervivum holo chrysum", apuntado en Icod el Alto ('Ten.).-Otra especie (Sempervivum balsamiferum) se llama en Lanz. alfarroba o farroba (M. Steffen, RH., año I95I, pág. 2I). Según Steffen serfa nuestra palabra idéntica con la española algarroba 'fruto de la Cratonia siliqua'.

*ambracásaca 'cabra de color pardo con manchas blancas', según Alvarez Delgado, en El Hierro ( $R H, \mathrm{XII}, \mathrm{I} 6 \mathrm{r}$ ). Con otra acentuación según los materiales de Armas Ayala: ambracasáca "canelosa con lunares blancos por todo el cuerpo' (TP, pág. 53). Mis fuentes del Hierro me dan embracasáca (con acento secundario en la primera a) "oveja de color canelo en la parte anterior y blanca en la parte posteriorn.

*ambracafiranca 'oveja blanca de medio atrás y firanca (véase) por delante', según Alvarez Delgado ( $R H, \mathrm{XII}, 282$ ). Falta en la colección de Armas Ayala sobre nombres dados por los pastores herreños a sus ganados (TP, pág. 53). Mis informadores del Hierro me dan embracafiránca (con acento secundario en la primera a) "oveja blanca por detrás y de color gris por delante».

*arija 'lluvia muy fina' en Arrecife (Lanz.); en La Gomera jarija, voz probablemente idéntica al esp. harija "polvillo blanco que el aire levanta de la harina cuando se ciernel, can. jarijo "puñado de gofio en polvo" en Pájara (F.) y "cantidad de gofio que se puede coger con los dedos" en Agulo, Valle Gran Rey y Hermigua (Go.), que tienen origen romance (*FARICULA).

arrife 'tierra sin cultivo' según Armas Ayala (TP, página 64), "terreno malo, inculto, pedregoso» según Alvarez Delgado ( $\left.R H, \mathrm{XII}, \mathrm{I}_{5} \mathrm{G}\right)$ en El Hierro. Mis informadores me 
dan esta palabra no sólo para El Hierro, sino también para Güimar (Ten.), Gáldar (GC.) y La Gomera. Según Régulo Pérez es usual, en Garafía (P.), arrifal, voz conocida también a mi informador de Gáldar (GC.). Vive la voz arrife también en las Azores iténue camada de terreno, em que aparecem, aqui e ali, cabeçotes de rocha subyacente (Figueiredo, Novo Dicion. I, 256). La voz es de origen árabe, idéntica al magrebi rif, y procede del latín ripa; véase $M$. $L$. Wagner en Biblos X, I934, 32.

*asaigo 'clase de arbusto siempre verde', "Rubia fruticosan en Icod, Realejo, Puerto de la Cruz (Ten.). Véase tadaigo. baifo 'cabrito', voz común, según mis fuentes, a todas las islas del Archipiélago cauario. Tenemos recogida para Gáldar (GC.) también la forma bafio. En una curiosa acepción, según Régulo Pérez (RH, XIII, I947, 253), se emplea baifo en Garafia (P.): papas baifas ipatatas que pareciendo buenas por fuera están podridas por dentron.-No tiene nada que ver con baifo la voz gaifo; véase galpo.

*balango 'avena loca', «Avena fatua en Güimar, San Andrés (Ten.), El Paso (P.), La Gomera y El Hierro. De La Palma he recogido también malango.-El doctor Steffen me recuerda el port. balanco 'erva nociva que cresce por entre as searas' (Figueiredo). Por lo tanto, seguramente no indígena.

*balo 'clase de arbusto parecido a un pino', "Plocama pendulay, en Taco, San Andrés (Ten.) y La Gomera. En Güimar (Ten.) he oído la forma bale.

*bea nombre genérico de "Sempervivum" en Agulo, Hermigua, Valle Gran Rey (Go.).

*bejeque, beheque (Ten.), en el Valle de Igueste bequeque 'Sempervivum canariense' según Steffen ( $R H, \mathrm{IX}, \mathrm{I} 943, \mathrm{I} 40$ ). Mis informadores me han dado bejeque para La Palma y La Gomera. En Tegueste y Taganana (Ten.) he oído bejeque al lado de bequeque.

belete (Go.), beleté (Ten.) 'calostro' según Alvarez Delgado ( $R H$, IX, I943, I36). En Tacoronte (Ten.) se usa beletén. Mis fuentes confirman la existencia de belete para La Palma, La Gomera, Lanzarote, Fiuerteventura y Hierro. En Gran Ca- 
naria es más común beletén. En La Palma se conocen belete y beletén. L a voz falta en las colecciones de Lugo y de Armas Ayala. Millares (pág. I9) anota beletén (primera leche que dan las reses después del parton.

*beril 'roca sobresaliente' en El Pinar y Ia Frontera (Hierro).

* berode o beroe en Santa Cruz (Ten.), berode en Güimar (Ten.), berode también en La Palma, La Gomera, El Hierro; Fuerteventura, berol en Icod el Alto ('Ten.), Agaete (GC.) y Lanzarote, bcrole en Gáldar (GC.). Así se llama un arbusto algo parecido a la tabaiba: es la 'Kleinia neriifolia'. La colección de Armas Ayala (TP, pág. 65) nos da berode sólo en el sentido secundario "dícese de la persona de carácter agriadon.

* bimba 'roca suelta' en Hierro según Armas Ayala (TP, página 65) y Alvarez Delgado ( $R H, \mathrm{XII}$, I57). Se usa, según nuestras referencias, en La Palma, La Gomera y El Hierro

*cairamo, cairano, cairón en los montes de Anaga (Ten.) 'zurrón de pellejo de cabra, especie de alforja donde transportan las herramientas, azadas y otros instrumentos' según Alvarez Delgado ( $R H, \mathrm{X}, \mathrm{I} 944$, I55). En Güimar (Ten.) he apuntado cairán "odre de cabra donde se transporta la leche».

calitofe véase algaritofe.

*carabuco 'vasija de barro de forma típica que sirve para ordeñar la leche’ (sic) en Chipude (Go.) según A. Mederos Sosa (TP, pág. I92).

*carisco 'laurel o viñátigo' según Alvarez Delgado ( $R H$, XII, I59) en Hierro. Mis informadores de esta isla (Mocanal, La Frontera, El Pinar) me dan la voz con otra acentuación: cárisco.

cocho véase gocho. .

* cómbaca o cónvaca cabra de color canela clara con rayas rojas en cabeza y patas' conforme a Alvarez Delgado ( $R H$, XII, I6I), combaca "canela clara con cabeza pintada como las patas en rayas de bermejo canelon según Armas Ayala (TP, pág. 53). Mis informadores del Hierro (E1 Pinar, Mo- 
canal, La Frontera) me dan oveja cómbaca "con el vientre color ceniza y el lonio color canelaw.

*creses 'bellotas o frutos del haya' según Alvarez Delgado ( $R H, \mathrm{XII}, \mathrm{I59}$ ) en Hierro. Conocen esta voz mis fuentes de La Gomera (Agulo, Valle Gran Rey, Hermigua); se usa con género femenino la crese o la cres (Valle Gran Rey) en el sentido de ufruto del haya», 'Myrica faya'. También en Tenerife se entiende bajo creses la fruta de la "Myrica faya".

"chajasco 'clase de escalera para transporte de un herido' según Alvarez Delgado en Güimar ( $R H$, VIII, 244). La voz no the ha sido confirmada en mis encuestas hechas en Güimar. Según J. Alvarez Delgado ('Teide, I945, pág. 30) representaria chajasco de uso actual en Canarias 'yerto, entesado, muerto' (completado por M. Steffen).

*chaboco 'cavidad natural en la lava para sembrar árboles' en Lanzarote, según Alvarez Delgado ( $R H$, VIII, I2). Parece la misma voz chajoco "huerto pequeño" dada para $\mathrm{El}$ Hierro en la colección de Armas Ayala (TP, pág. 66).

chajinaste véase tajinaste.

* chaóra en Icod el Alto ('Ten.), chajora en Güimar (Ten.), chahorra en Gáldar (GC.), nombre genérico de "Leucophae», sobre todo "L. candicans" y "L. argosphacelus".

*charasco 'trozo de ramo seco' en Agulo, Valle Gran Rey y Hermigua (Go.), charamusco con el mismo sentido en Mocanal, El Pinar, La Frontera (Hierro). Confróntese charamusca 'leña menuda con que se hace fuego en el campo' en el español de América (DMLE).

*cheire 'lluvia muy fina' en Garafía (P.), según informe de Régulo Pérez. La palabra la relaciona José Pérez Vidal con el port. cheiro 'olor' (RDTP, V, I949, I87). Esto se me antoja poco probable.

chénique "cada una de las tres piedras que se colocan en el fogón isleño' (Ten.) según Alvarez Delgado ( $M G, I, 3 \mathrm{I}$ ). La colección de Arnias Ayala da, en la misma acepción, para Gran Canaria y Hierro (TP, pág. 76) tenique y tinique. De mis encuestas personales resulta chénique para Güimar (Ten.), chinique para Agulo, Valle Gran Rey y Hermigua (Go.), te- 
nique para Arrecife (Lanz.) y Pájara (F.), tinique para Mocanal, La Frontera y El Pinar (Hierro), siempre en el mismo sentido. İ̀ La Palma, según nuestras referencias, hay tenéque y en Gran Canaria tenique en el sentido de 'trozo de piedra'. Enl la colección de voces de Gran Canaria de Millares se encuentra tenique 'pedazo de lecho (¿leño?) cortado y dispuesto para encender y alimentar el fuego' (pág. 153).

*chocalla 'oveja bermeja oscura con pintas blancas' conforme a Alvarez Delgado (RH, XII, 282). Para Hierro (NOcanal, La I'rontera, El Pinar) me confirman chocalla en el sentido de «oveja de color ceniza con cabeza blanca».

chuchango 'caracol' en Gran Canaria (Millares, pág. 84). Voz registrada en la misma forma por las colecciones de Lugo y de Armas Ayala. Mis informadores de Güimar (Ten.) y de La Gomera me dan, en la misma acepción, chuchanga.

*csmira 'corteza del pino' en Hierro, según Alvarez Delgado (RH, XII, I55). Mis fuentes de Mocanal, La Frontera y El Pinar confirman esmira 'cáscara seca del pino'.

*eres m. "hoyo o poceta formados en las rocas impermeables del álveo de los barrancos donde se acumula con el agua de lluvia arena fina' ('Ten.), según Alvarez Delgado ( $R H$, VII, 2I0). He recogido en Güimar un ere y, en boca de los jóvenes, un lere. La voz, sin duda, es idéntica a herez "cisterna', recogida de la lengua de los guanches y usada por Juan de Abreu en su "Historia de la conquista de las siete islas de Gran Canaria" (I632).

* firánca o filánca "cabra de color gris oscuro o revuelto de blanco y negro', según, Alvarez Delgado en Hierro ( $R H$, XII, I6I); firanca "canelosa oscura", conforme a Armas Ayala (TP, pág. 53). Voz confirmada por mis informadores del Hierro: firánca 'cabra u oveja de color gris oscuro'.

* gainás 'un poco de gofio amasado y cogido entre los dedos' en T'enerife, según Alvarez Delgado ( $M G$, I, págs. I6 y 30). En Güimar ('Ten.) he recogido gainás 'un puño de gofio amasado'.

* galpo en Agulo, Valle Gran Rey y Hermigua (Go.), garpo en Mocanal, La Frontera y El Pinar (Hierro) 'animal a 
quien falta un testículo'. En La Palma se usa, según Régulo Pérez, en el mismo sentido gaifo, gaifito, galpito o gaipito ( $R H$, XIII, 253).-Esta voz no tiene nada que ver, como presume Régulo Pérez, con baifo kcabriton.

gamame y agamame 'puñado de gofio en polvo que se toma como entremés con vino', según Armas Ayala, en Hierro (TP, página 69). Según Alvarez Delgado también en La Gomera, mientras que en Fuerteventura se dice gagame ( $R H, \mathrm{IX}$, I46). Para Hierro he recogido ganname 'puñado de gofio en polvo'.

* ganneita '(cabra) de color blanco con pintas bermejas', Alvarez Delgado ( $R H, \mathrm{XII}, 282$ ) en $\mathrm{El}$ Hierro. Mis informadores de Mocanal, La Frontera y El Pinar me han dado ganita "cabra con el vientre gris y el lomo color canela" (corresponde a la oveja cómbaca).

gánigo 'vasija de barro, cazuela pequeña, lebrillo' en 'Tenerife y Gran Canaria, según las colecciones de Millares, Lugo y Reyes.

"gasia 'especie de cítiso', "Cytisus stenopetalus" en La Palma. Véase tagasaste.

* gavia en Agulo, Valle Gran Rey y Hermigua (Go.) 'punta más alta de un árbol'; en Mocanal, La Frontera y El Pinar (Hierro) 'vara de árbol'; en otros sitios de la Gomera 'larga rama de higuera'.-Idéntico con el esp. gavia 'cofa de las galeras' (según Steffen).

gocho y cocho 'trozo de terreno o huerta', conforme a Alvarez Delgado ( $R H, \mathrm{X}, 20)$. En Güimar (Ten.) he recogido gocho 'pequeño trozo de terreno'.

gofio 'harina gruesa de maiz, trigo o cebada tostada' (originariamente sólo de cebada tostada), voz común a todas las islas del Archipiélago. Se usa también en Madera y en muchos países de América: Argentina, Bolivia, Cuba, Ecuador, Puerto Rico.

* goga 'pequeña cantidad de gofio en polvo que se coge con dos dedos'. Tenemos recogida esta voz para Güimar (Ten.).

goro 'establo para cabras, cerdos u ovejas', según Alvarez Delgado ( $R H$, VII, I84); según Armas Ayala (TP, pág. 69) 
'cueva natural utilizada por los pastores como refugio', en Hierro. En Gran Canaria goro se refiere también a la "cueva donde se almacena el barro de los alfareros" ( $T P$, pág. I76). He recogido, en el sentido de 'pocilga rudimentaria', goro para Güimar ('Ten.), La Palma y. Lanzarote. En El Hierro se usa, según Armas Ayala (TP, pág. 69), gorete "muralla hecha de cañas, maderos o cualquier otra materia que sirve para proteger un plantío contra el vienton.

*guanil 'salvaje, libre, sin dueño, no marcado' en Lanzarote, según Alvarez Delgado ( $R H$, VIII, Io). Mis informadores del Hierro (Mocanal, La Frontera, El Pinar) y de Fuerteventura (Ampuyenta) confirman guanil con la acepción de 'no marcada' (cabra, oveja). Véase juanil.

* guargaso en San Andrés (Ten.), cualgaso en Güimar (Ten.) 'jara negra', "Cistus monspeliensis»; voz idéntica al esp. jaguarzo, valenc. xagıar ço 'clase de jara' ("Cistus"). Voz de origen árabe šaqwâs (Steiger, pág. 215).

* guásimo, guácimo, guársamo cuenco o bueco de las gruesas ramas de árboles, abiertas para recoger en ellas el agua destilada por las ramas durante las nieblas frescas' en Hierro, couforme a Alvarez Delgado (RH, XII, I56). He recogido para Mocanal, La Frontera y El Pinar (Hierro) guásamo "concavidad de un árbol donde se mantiene el agua».

*guinso 'tambor' en Hierro, según Alvarez Delgado ( $R H$, XII, I55). Esta voz no me ha sido confirmada por ninguno de mis informadores de Hierro, ni tampoco de las otras islas.

guirre 'buitre' en Gran Canaria (Millares, pág. 76). He podido recoger esta voz también en Güimar (Ten.); voz conocida también a mis fuentes de La Gomera y de Hierro.

*inganso 'raspa que queda del racimo de uvas', según mis informadores de La Gomera y del Hierro. Lugo, en su Colección, nos da engazo "ramajo de racimo de uvas», voz idéntica a la port. engaço que tiene la misma acepción.

*irama (Hierro), orijama (Gomera) 'especie de terebinto" (Alvarez Delgado, RH, XII, I59). Me confirman irama para Hierro, orijana para La Gomera como nombre de un arbus- 
to de flores amarillas. Se refiere, según Steffen, a "Cneorum pulverulentum".

*jaicán 'variedad de orchilla, planta tintórea' en La Gomera (Alvarez Delgado, $R H, \mathrm{XII}, \mathrm{I} 60$ ). Voz desconocida a mis informadores de La Gomera y de Hierro. Según me informa Steffen, la voz existe realmente en La Gomera; véase también Mederos Sosa, RH, XII, 442. Es la uparmelia perlata Ach.».

*jameo 'cueva grande y muy profunda' en Lanzarote, según Alvarez Delgado ( $R H$, VIII, II).

*jarija, jarijo, véase arija.

*jirdana 'especie de retama', según todos mis informadores de Ia Gomera y de Hierro. Steffen me informa que gilda$n a$, en Tenerife, es el "Cytisus canariensis"; en Gran Canaria gildana de la cumbre "Cytisus congestus".

*jórana "cabra de color negro y con la barriga bermeja - rojo dorada' en Hierro (Alvarez Delgado, RH, XII, I6I). Según mis fuentes del Hierro (Mocanal, La Frontera, El Pinar) jórana se refiere a la "oveja de lomo negro y de vientre amarillento'. En la colección de Armas Ayala se da, con acentuación errónea, jorana 'negra y bermeja toda la barriga' (TP, pág. 53).

*juanil 'único, sencillo' en Lanzarote (Alvarez Delgado, $R H$, VIII, Io). Voz probablemente idéntica a guanil (véase).

* juercan véase ajergo.

* malango véase balango.

*majo o maho 'calzado de pellejo crudo' en Hierro (Alvarez Delgado, $R H, \mathrm{XII}$, I64). Me confirman esta voz para Hierro.

*manajáis, manajáise 'cabra negra con lunar o mancha blanca en la cabeza' en Hierro, según Alvarez Delgado ( $R H$, $\mathrm{XII}, \mathrm{I62}$ ). Mis informadores de Hierro me dan manajáise 'oveja negra que tiene la cabeza blanca'.

* mástuca "cabra de color revuelto de blanco y negro con predominio de blanco' en Hierro (Alvarez Delgado, $R H, \mathrm{XII}$, I62). Según mis informadores de Hierro, mástuca se refiere a la 'oveja de color castaño'. In la colección de Armas Ayala, 
con acentuación errónea: mastuca "canelosa oscura, o sea, un color revuelto de blanco y negro' (TP, pág. 53).

* mérusa 'cabra u oveja blanca y canelosa, o bermeja con pintas blancas' en Hierro, según Alvarez Delgado ( $R H, \mathrm{XII}$, páginas $I 62$ y 282). Según mis fuentes del Hierro mérusa se refiere a la 'oveja con manchas de un canelo muy fuerte'.

*ómana o hómana "cabra de un color blancuzco o blauquecino' en Hierro (Alvarez Delgado, RH, XII, I62). Mis informadores de Hierro me han dado omana 'oveja de color gris con cabeza blanca'.

*orijama véase irama.

pambufo véase tambufo.

*pantana 'especie de calabaza' en La Gomera y Mocanal (Hierro).

perinquen 'especie de lagarto' en Gran Canaria (Millares, página II6), perenquén 'lagarto amarillo o salamanquesa' (Lugo, pág. I4I). In mis encuestas personales he obtenido perenquén para Santa Cruz de Tenerife y Tacoronte (Ten.), perinquén para Güimar (Ten.), Lanzarote y Fuerteventura, perinquel para Gáldar y Valleseco (GC.), perinqué para Agaete (GC.), prácan para Agulo (Go.), plácan para Valle Gran Rey y Hermigua (Go.). En Hierro se usa la forma perenquén. El nombre no se refiere ni al lagarto ni a la salamanquesa, común (de agua), sino al animal que, en francés, se llama "gecko". Es el "Ascalabotes (Platydactylus, Tarentola) Delalandii» y "Ascalabotes (Platydactylus, Tarentola) Boettgeri».

*petequén 'trozo de piedra' en Agulo (Go.).

* pincora 'cima de un árbol' en Breña Alta (P); en la misma acepción piringola en Santa Cruz de La Palma.

*pipana "cabra de varios colores o blancazca con manchas oscuras en las quijadas' en Hierro (Alvarez Delgado, $R H$, XII, I62). Tengo recogido de mis informadores de Hierro $p i-$ pana 'oveja pintada de canelo y blanco'. En la colección de Armas Ayala con acentuación errónea pipana.

*plácan, prácan, véanse perinquén.

*sanjora 'nombre de un árbol o de una mata' en Hierro (Alvarez Delgado, RH, XII, I59). Mis informadores de Hie- 
rro me dan esta voz como nombre de una especie de siempreviva, planta crasulácea, que se encuentra en las paredes de los peñascos'.

*sorejona "de cabra u oveja que tiene las orejas muy pequeñas' en Mocanal, I a I'rontera y El Pinar (Hierro).

sorimba en Güimar (Ten.), surimba en La Laguna ('Ten.) "lluvia muy fina». En La Palma hay sorimbar 'lloviznar'. El léxico de Millares (pág. I47) apunta sorimba «miedo, vergüenza, cortedad, frion.

tabaiba, nombre que se da a toda clase de los ueuforbios, titímalo o lechetrezna». Voz común a todas las islas del Archipiélago. La forma tobaiba en la colección de Lugo (página I59) debe de ser error por tabaiba. - La palabra se emplea también en el portugués de Cabo Verde: tabaibo 'figueira -do- inferno' (Figueiredo).

*tabaraste 'ajo silvestre' en el P'aso (P.).

tabefe 'suero del queso' en Gran Canaria (Millares, página I49). En la misma acepción me ha sido confirmado para La Gomera, Garafía (P.) y varios pueblos de Gran Canaria. En Arucas (GC.) y Valleseco (GC.) es usual la forma tabique. En Agaete (GC.), al lado de tabefe se usa también tabumbe. La voz que pertenece también al portugués (labefe 'suero de leche') es de origen árabe: tabix (Steiger, pág. r50).

*tabobo al lado de jabobito, abobito 'pájaro conocido en los campos con el calificativo de lavandero por posarse en los sitios húmedos' en Gran Canaria (Armas Ayala, TP, página 62). He recogido personalmente tabobo, al lado de abobito, para Güimar, tabobo para La Palma, Gomera y Lanzarote, jabobo para El Paso (P.), jabubo para Mocanal, La Frontera y El Pinar (Hierro). Es, en todos sitios, en nombre de la 'abubilla' (Upupa epops). En árabe magrebi, el mismo pájaro se llama thêr abäbil. Es claro el origen onomatopéyico comprobado por apupii, nombre del mismo pájaro que tengo recogido en Gran Canaria.

*tabona 'piedra azufrosa' del Teide (Ten.), según. Armas Ayala (TP, pág. 75). Esta voz, según nuestras referencias, está viva también en El Hierro. ¿Tiene que ver algo con la 
palabra tabón que el Diccionario manual de la Acadenia Española registra, en la acepción de 'terrón', para Burgos y Palcncia?

*tabona 'antiguo molino puesto en marcha por un burro', en Breña Alta (P.).

*tacanija 'harina de raíz de helecho que sirve para preparar una especie de gofio' en La Gomera, según A. Mederos Sosa (TP, pág. I92). Conforme a nuestras referencias 'polvillo de gofio' en Agulo, Hermigua y Valle Gran Rey (Go.). En otros sitios de La Gomera se usa tacanijo 'puñado de gofio en polvo'.

*tadaigo 'nombre de una mata' en El Hierro (Alvarez Delgado, RH, XII, I59). Mis informadores de Hierro me dan tadaigo como nombre de la mata que en 'lenerife se llama asaigo "Rubia fruticosa".

*tafcina 'maiz tostado' en Güimar ('I'en.), Gomera, Hierro, İuerteventura.

*tafor 'calostro, primera leche que da la hembra' en Icod (Ten.). En Güimar se llama tafosa, en Valle Seco ('Ten.) leche tafosa.

*tafuriaste 'tambor' en Tenerife, según Alvarez Delgado (RH, XII, I56). Voz desconocida a mis informadores de Gran Canaria, La Palma, Gomera, Hierro y Fuerteventura.

*tagasaste 'especie de cítiso'. "Cytisus proliferus", según mis fuentes de Tenerife, La Palma, Gomera y Hierro. Véase gasia.

taifa 'concurso o reunión' en Gran Canaria (Millares, página 149). Mis informadores de Pájara (F.), Agaete (GC.) y Valleseco (GC.) me dan taifa en la acepción de cada turno de baile'. Según Millares es voz moruna, importada por los marinos de la costa de Africa.

*tajaraste 'baile típico canario' (Armas Ayala, TP, página 75). Me han confirmado esta voz mis fuentes de Tenerife, La Palma, Gomera y Hierro.

*tajinaste "nombre de varias especies de "Echium» de la familia de las borragináceas (Echium strictum, E. fastuosum, E. giganteum)', en La Laguna, Güimar, Icod (Ten.), 
Agulo (Go.). En San Andrés he recogido la forma chajinaste; uno de mis informadores de Hierro me ha dado tajinasque. En La Palna, según Steffen (RH, XIII, I90), también tainaste.

*tamailmna o tannaisma 'aguzanieves', según mis referencias de Agulo, Valle Gran Rey y Hermigua (Go.).

*tamarco o tamargo "balde o zurrón de cuero para extraer agua de los aljibes' (Alvarez Delgado, RH, XII, I57), tamarco «balde de cuero o madera en E1 Hierro, según Armas Ayala (TP, pág. 75). Esta voz es desconocida a mis fuentes del Hierro y de Ia Gomera. Las fuentes históricas nos dan tamarco en el sentido de 'capa de piel'.

*tambufo 'gordo y bajo', 'regordete', según mi fuente de Agulo (Go.). En Gran Canaria se dice pambufo (Millares, página II2 y Armas Ayala, TP, pág. 73). In esta misma forma me ha sido confirmada la voz para La Palma, Lanzarote $y$ Fuerteventura. En otros sitios de Gran Canaria y La Palma se dice también gordufo.

*tapagán 'especie de lechuza' en Agulo, Hermigua y Valle Gran Rey (Go.), también en Garafía (P.).

teneque, tenique véanse chenique.

*tolda "especie de euforbia, "Euphorbia aphylla", planta muy baja sin hojas que forma una almohada redonda', en. Tenerife, según M. Steffen (RH, X, I944, I42). Voz desconocida a mis informadores de La Gomera y del Hierro.

Después de haber sido escrito este trabajo en el verano dé I95I, he tenido ocasión de conocer el tratado de ERNST ZyHLARZ: Das kanarische Berberisch in seinem sprachgeschichtlichen Milien [El beréber canario en su medio histórico-lingiiistico ]. De: Zeitschrift der Deutschen Morgenzländischen Gesellschaft, tomo roo, I950, pág. 403-460) ${ }^{1}$. La teoría muy

1 Doy aqui el juicio que publiqué en las reseñas del Archiv für das Studium der neueren Sprachen, tomo 189, 1953, pág. 406:

Aunque sea éste un tema propio de 'africanistas' roza cuestiones. no desprovistas de importancia para el hispanista. Un notable espe- 
atrevida sustentada alli de un sustrato múltiple racial y lingüístico de las islas Canarias no se confirma bien por nuestra investigación de las palabras canarias. De nuestros materiales más bien hay que obtener la inupresión de un sustrato lingüístico bastante unitario: de las palabras tratadas en nuestro trabajo, catorce se hallan en dos islas, siete en tres islas, seis en cuatro islas, tres en cinco islas, tres en seis islas, cinco en siete islas. Sólo la isla de Hierro se nos manifiesta por un número considerable de elementos que son desconocidos a las demás islas: ambracasaca, ambracafiranca, beril, càrisco, còmbaca, chocalla, esmira, firanca, gamita, guàsamo, jòrana, majo, manajàise, màstuca, mérusa, òmana, pipana, sanjora, sorcjona.

\section{GERIAARD ROHLFS.}

cialista de las lenguas aborigenes norteafricanas examina criticamente el material lingiústico canario que nos ha llegado por tradición escrita y demuestra como muy problemática la fijación de su fonética y ortografia; a la vez califica de diletante e ilusoria la opinión muy extendida de que el canarı tenga el valor de una fuente lingüística proto-líbica. En realidad hemos de admitir, para el Archipiélago canario, condiciones lingüísticas mucho más variadas: en Gran Canaria, como resto de colonizadores primitivos europidas rubios y ojiazules (celtiberos?), una lengua de carácter indogermánico; en Fuerteventura $y$ Lanzarote la lengua de la antigua Mauritania; en Tenerife otra lengua al parecer aglutinante de 'habitus' desconocido; en Hierro una lengua neopúnica, y en La Gomera, Palma y La Gomera beréber (=neolibica). - Esta atrevida teoria rica en puntos de vista y expuesta con gran seguridad (ital vez excesiva?) obliga a replantear a foudo todos sus problemas. - Contra la complejidad de las condiciones lingüisticas supuesta por $Z$. puede hacer algunas objeciones taunbién un aficionado absoluto 'in rebus africanis', p. e.: las palabras de substrato que se conservan aún hoy en el español canario y que Z. no tiene en cuenta son, la mayor parte de las reces, comunes a varias islas: belete "calostro" (GC, $P, \mathrm{~L}, \mathrm{~F}, \mathrm{G}, \mathrm{T}$ ); chinique (G), tinique (F), tenique (GC), tenćque (P) "piedra del hogar"; perinquén ( $T, F, L)$, perenquén (IF), prácan (G) "salamanquesas; tabaiba (T, P. G, GC, I, F) "especie de euforbia", etc. Al sostener una teoria tan revolucionaria no pueden ser ignorados tampoco los topónimos de las diferentes islas tau caracteristicos en su consonancia fonétican. 